\title{
Cytogenic and molecular studies of male infertility in cases of $Y$ chromosome balanced reciprocal translocation
}

\author{
DING WANG ${ }^{1,2^{*}}$, RUI CHEN ${ }^{1,2^{*}}$, SHU KONG ${ }^{1,2}$, QIAN-YING PAN ${ }^{1,2}$, YU-HONG ZHENG ${ }^{1,2}$, \\ WEN-JUN QIU ${ }^{1,2}$, YONG FAN ${ }^{1,2}$ and XIAO-FANG SUN ${ }^{1,2}$ \\ ${ }^{1}$ Key Laboratory for Major Obstetric Diseases of Guangdong Province and \\ ${ }^{2}$ Key Laboratory of Reproduction and Genetics of Guangdong Higher Education Institutes, \\ The Third Affiliated Hospital of Guangzhou Medical University, Guangzhou, Guangdong 510150, P.R. China
}

Received April 5, 2016; Accepted March 7, 2017

DOI: $10.3892 / \mathrm{mmr} .2017 .6835$

\begin{abstract}
Y-autosomal translocation has been previously reported in association with male infertility; however, the mechanisms of Y-autosomal translocation and non-obstructive azoospermia or severe oligospermia remain unclear. G-banding and fluorescence in situ hybridization (FISH) were performed to analyze the translocation of chromosomes, and a single nucleotide polymorphism (SNP) genotyping assay was used to test mutations. The present study describes three new cases with a de novo balanced translocation $\mathrm{t}(\mathrm{Y} ; 13), \mathrm{t}(\mathrm{Y} ; 9)$ and $\mathrm{t}(\mathrm{Y} ; 6)$. To further explore the genotype-phenotype correlation, G-banding and FISH were performed and indicated the presence of a derivative chromosome. The SNP genotyping assay using a microarray revealed no abnormality, especially in the $\mathrm{Y}$ chromosome. Molecular deletion analysis demonstrated that no microdeletion was detected in the azoospermia factor region of the $\mathrm{Y}$ chromosome in the examined, infertile men. Based on these observations, the authors proposed the hypothesis that a position effect involving unknown spermatogenesis regulatory gene(s) serves a key role in male infertility.
\end{abstract}

\section{Introduction}

Male infertility can be caused by several genetic factors, including chromosomal abnormalities, Y chromosome microdeletions and gene mutations (1). Cytogenetic anomalies, especially structural chromosomal aberrations are

Correspondence to: Dr Xiao-Fang Sun, Key Laboratory for Major Obstetric Diseases of Guangdong Province, The Third Affiliated Hospital of Guangzhou Medical University, 63 Duobao Road, Liwan, Guangzhou, Guangdong 510150, P.R. China

E-mail: xiaofangsun@gzhmu.edu.cn

*Contributed equally

Key words: Y-autosomal translocation, fluorescence in situ hybridization, single nucleotide polymorphisms, microdeletions an important cause of male infertility (2). Deletion of the azoospermic factor on the long arm of the $\mathrm{Y}$ chromosome is known to be involved with spermatogenesis defects (3). Cystic fibrosis genes mutations have previously been reported in male infertility (4).

Y-autosome translocations are rare in humans, and they may be identified in both fertile and sterile males (5). The rate of chromosomal rearrangement ranges from 10-15\% in azoospermic males (6). The frequency of Y-autosome translocations in the general population is approximately 1 in 2,000 (7,8). However, previous studies mainly basing G-banding with little analysis on the etiology of fertility (9-13).

In the present study, a whole genome SNP microarray and a genetic analysis of the $\mathrm{Y}$ chromosome with 20 sequence-tagged sites were performed on three adult azoospermic male with de novo Y-autosome translocations. The etiology and clinical features of this rare disease were briefly discussed.

\section{Patients and methods}

Cases. The first patient (P1) was a 34-year-old man with a balanced translocation $t(Y ; 13)(q 12 ; q 21)$ (Fig. 1A). His wife possessed a normal karyotype. He was a well-developed male weighing $79 \mathrm{~kg}$ with a height of $175 \mathrm{~cm}$. Physical examinations revealed normal adult pubic and axillary hair. The penis, epididymides, spermatic cords and prostate were normal. The plasma levels of lactate dehydrogenase, follicle stimulating hormone, prolactin and testosterone were within normal limits. Repeated semen analyses indicated azoospermia.

The second patient (P2) was another 34-year-old man with a balanced translocation $\mathrm{t}(\mathrm{Y} ; 9)(\mathrm{p} 11.2 ; \mathrm{q} 21)$ (Fig. 1B). The patient, who was phenotypically normal and had normal clinical examinations, was ascertained for infertility. Sperm counts presented severe oligozoospermia azoospermia. The wife of P2 was able to conceive naturally once but spontaneous abortion occurred.

The third patient (P3) was a 26-year-old man with a balanced translocation $\mathrm{t}(\mathrm{Y} ; 6)(\mathrm{q} 12 ; \mathrm{q} 21)$ (Fig. 1C). He was a well-developed male weighing $65 \mathrm{~kg}$ with a height of $170 \mathrm{~cm}$. His physical examination was normal, and had an unremarkable family history. No sperm were identified in any of the three routine semen analyses. 
A

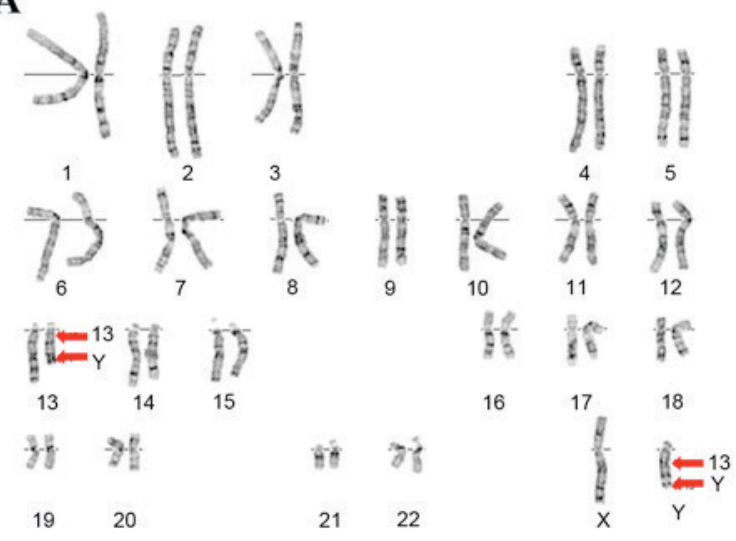

B

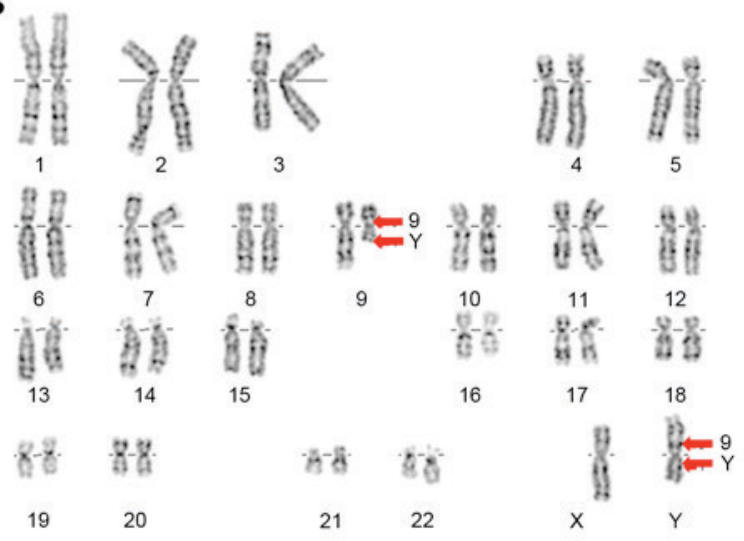

C

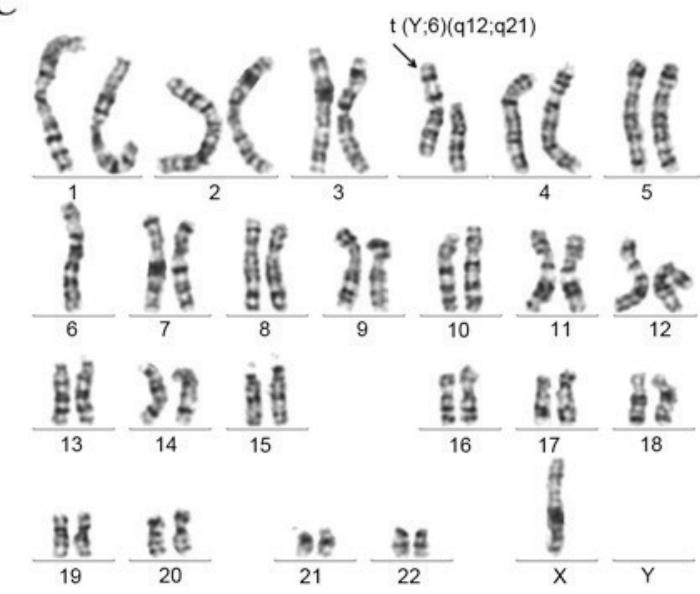

D

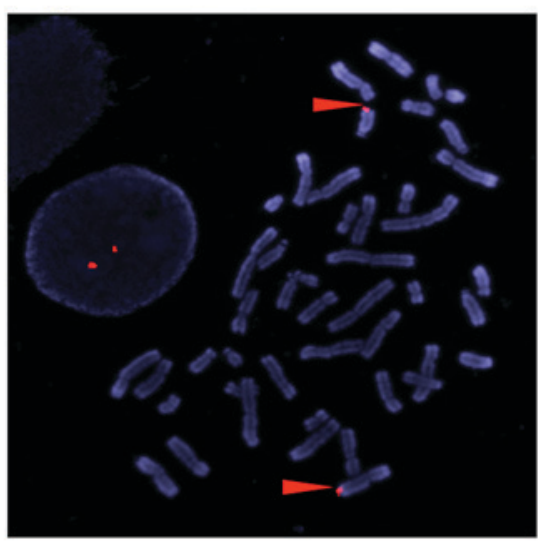

Figure 1. (A) Metaphase spread of P1 following G-banding. (B) P2 G-banding. (C) P3 G-banding. (D) Fluorescence in situ hybridization with probes of the chromosome Y (shown in red) on blood lymphocytes of P1. P, patient.

Ethical approval. All procedures performed in studies involving human participants were in accordance with the ethical standards of the ethics committee of The Third Affiliated Hospital of Guangzhou Medical University (Guangzhou, China) and with the 1964 Helsinki Declaration and its later amendments or comparable ethical standards. All patients gave their informed consent to participate in the present study.

Karyotype. Cytogenetic investigations were performed on the patients' chromosomes obtained from peripheral blood lymphocytes. The lymphocytes were cultured for $72 \mathrm{~h}$ in RPMI medium 1640 (Gibco; Thermo Fisher Scientific, Inc., Waltham, MA, USA), phytohemagglutinin (Shanghai Yihua Medical Science \& Technology Co., Ltd., Shanghai, China), and fetal bovine serum (Beijing Dingguo Changsheng Biotechnology Co., Ltd., Beijing, China) following treatment with $50 \mu \mathrm{g} / \mathrm{ml}$ colcemid (Sigma-Aldrich; Merck KGaA, Darmstadt, Germany). The cells were made hypotonic using potassium chloride $(0.075 \mathrm{M})$, fixed in a fixation medium [acetic acid combined with methanol (1:3)] at room temperature for $1 \mathrm{~h}$, dropped on precooled glass and incubated at $60^{\circ} \mathrm{C}$ overnight. Metaphase chromosome spreads were studied by standard G-banding procedures, using trypsin and Giemsa for G-banding. FISH analysis of the rearrangement of the human
$\mathrm{Y}$ chromosome was conducted using specific $\mathrm{Xq} / \mathrm{Yq}$ probes (Vysis; Abbott Pharmaceutical Co., Ltd., Lake Bluff, IL, USA).

Chromosome microarray analysis (CMA). CMA-SNP array analysis was performed on the probands' and their parents' peripheral blood samples using the Affymetrix Cyto HD Array (Affymetrix, Inc., Santa Clara, CA, USA). The DNA was amplified, labeled and hybridized to the CytoScan HD Array platform according to the manufacturer's protocol. The array was designed specifically for cytogenetic research. It includes $>2$ million markers across the genome, in addition to SNP probes and probes for detecting copy-number variations. The raw data files were obtained by scanning the arrays, which were then analyzed with the Chromosome Analysis Suite software 33.1 (Affymetrix, Inc.), and the reference genome GRCh37 (hg19) was used for the annotations. Only those signals meeting the manufacturer's quality cut-off criteria were included in the present analysis. Gains and losses that affected a minimum of 50 markers over $100 \mathrm{~kb}$ lengths were initially considered.

Genetic analysis of $Y$ chromosome. A total of 20 sites were analyzed with regards to the $\mathrm{Y}$ chromosome microdeletion by the Y chromosome Deletion Detection System kit (version 2.0; Promega Corporation, Madison, WI, USA). This system is 
Table I. Genotype-phenotype correlation in adult males with Y;13,Y;6 translocation.

\begin{tabular}{llllr}
\hline Karyotype & Origin & \multicolumn{1}{c}{ Molecular analysis } & Sperm count & (Refs.) \\
\hline $\mathrm{t}(\mathrm{Y} ; 13)(\mathrm{q} 11.2 ; \mathrm{q} 12)$ & $\mathrm{NP}$ & Interval 5L deleted & Azoospermia & $(14)$ \\
$\mathrm{t}(\mathrm{Y} ; 13)(\mathrm{p} 11: 32 ; \mathrm{p} 12)$ & de novo & Intact SRY and AZF deletion & Oligozoospermia & $(15)$ \\
$\mathrm{t}(\mathrm{Y} ; 6)(\mathrm{Yp} 6 \mathrm{p} ; \mathrm{Yq} 6 \mathrm{q})$ & $\mathrm{NP}$ & NP & Azoospermia & $(16)$ \\
$\mathrm{t}(\mathrm{Y} ; 6)(\mathrm{q} 11.23 ; \mathrm{p} 11.1)$ & $\mathrm{NP}$ & Retention of the DAZ gene & Oligozoospermia & $(17)$
\end{tabular}

NP, not performed; SRY, sex determining region Y; AZF, azoospermia region; DAZ, deleted in azoospermia.

A

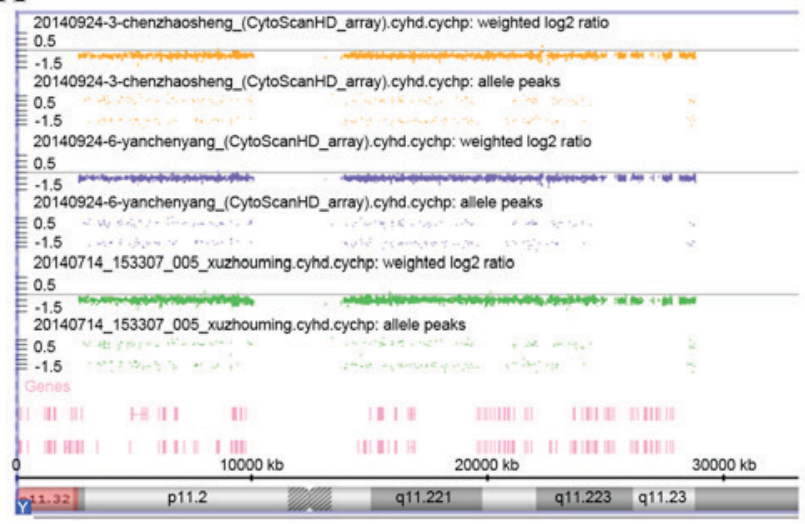

B

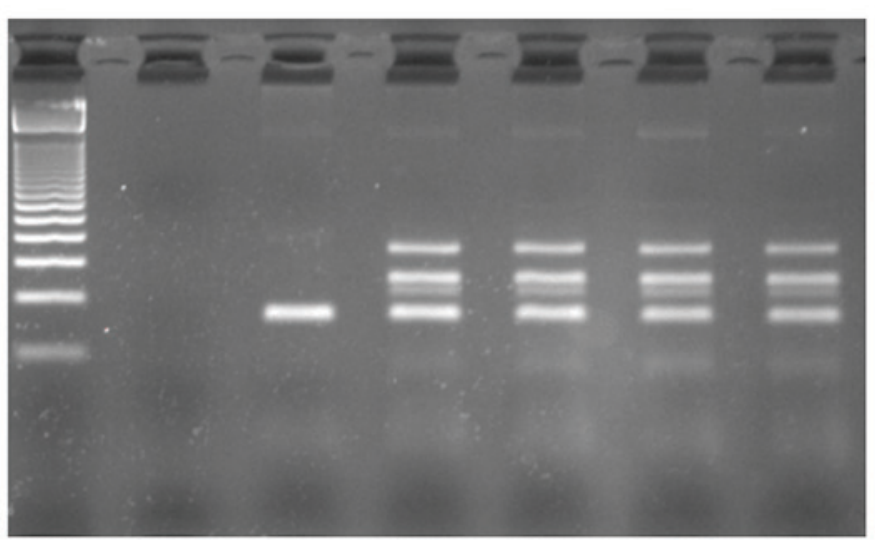

Figure 2. (A) Single nucleotide polymorphism array analysis. (B) Y chromosome microdeletion analysis (Multiplex D Master Mix). Lane 1, marker; lane 2, blank; lane 3, female; lane 4, normal male; lane 5, P1; lane 6, P2; lane 7, P3. P, patient.

designed to detect deletions occurring in the azoospermia factor region on the $\mathrm{Y}$ chromosome long arm (YqAZF). This system consists of 20 primer pairs that are homologous to previously identified and mapped sequence-tagged sites. The primers have been combined into five sets for use in multiplex polymerase chain reactions (PCR): Multiplex A Master Mix for the detection of deleted in azoospermia (DAZ; SY254), DYS240 (SY157), DYS271 (SY81), DYS221 (SY130) and anosmin 2, pseudogene (SY182) microdeletions, with lysine demethylase 5C (SMCX) as control; Multiplex B Master Mix for the detection of lysine demethylase 5D (SYPR3), DYS218 (SY127) and DAZ (SY242 and SY208) microdeletions, with SMCX as control; Multiplex C Master Mix for the detection of DYS219 (SY128), DYS212 (SY121), DYF51S1 (SY145) and DAZ (SY255) microdeletions, with SMCX as control; Multiplex D Master Mix for the detection of DYS223 (SY133), DYS236 (SY152) and DYS215 (SY124) microdeletions, with SMCX as control; Multiplex E Master Mix for the detection of sex determining region Y (SY14), DYS224 (SY134), DYS148 (SY86) and DYS273 (SY84) microdeletions, with zinc finger protein, $\mathrm{X}$ linked/zinc finger protein, Y-linked as control. The sequence tagged site number is presented in brackets. This makes it possible to determine the presence or absence of all 20 sequence-tagged sites by performing five parallel PCR amplifications.

\section{Results}

Cytogenetic studies. All the patients, their spouses and their parents had normal karyotypes based on G-banded metaphase chromosomes. $\mathrm{G}$ banding indicated that all metaphase cells revealed an apparently balanced reciprocal translocation (Fig. 1A). By performing FISH, the translocation between chromosome 13 and the Y chromosome were clearly defined in P1 (Fig. 1D). G-banding demonstrated an apparent Y autosomal translocation, and a derivative $\mathrm{Y}$ chromosome was observed in P2 (Fig. 1B). The presence of a whole chromosome translocation was revealed in P3 (Fig. 1C).

Molecular analysis. The SNP-array of three patients using the Affymetrix Cyto HD array did not reveal any abnormalities, especially within the Y chromosome (Fig. 2A). At a molecular level, by means of PCR, no microdeletions were detected in the AZF region of the $\mathrm{Y}$ chromosome in the infertile man (data not shown). Y chromosome microdeletion analysis performed by using the Y chromosome Deletion Detection system did not identify any deletion (Fig. 2B).

\section{Discussion}

Reciprocal translocations between the Y and a non-acrocentric chromosome, such as the de novo balance translocation described in the present study are rarely observed (17-20). A review of previous report concerning a Y-autosome carrier indicated that most of the translocations led to azoospermia or oligozoospermia (Table I), and the phenotype was associated with the localization of the breakpoint and the nature of the lost Yq material (21-23). In the current study, three new cases of balanced de novo $\mathrm{Y}$ autosomal translocation 
are described in infertile men. Our patients had normal development and normal phenotype besides presenting severe oligozoospermia. The cytogenetic G-banding analysis revealed $\mathrm{Y}$ autosomal translocation in all metaphase cells.

Since the sterile phenotype associated with Yq breakpoint localization and Yq deletion, it is of great importance to fully understand the Yq breakpoint by DNA molecular studies (22). However, in the present study, CMA-SNP array analysis and molecular deletion analysis didn't reveal any deletion/duplication in the patients, as well as microdeletions in the AZF region. This assignment and the retention of the DAZ gene could not explain the infertility of the patients. In addition, Y chromosome microdeletion demonstrated that no deletion occurred in any of the patients. On this basis, the authors propose the following hypothesis that a position effect of unknown spermatogenesis regulatory gene(s) serve a key role in male infertility and rearrangement of a chromosome can regulate gene expression without microdeletion in the AZF region or other conventional genetic factors. Alternatively, as a previous study reported, the translocation regulates gene expression by performing a disturbance to the heterochromatin region of chromosomes (5).

In conclusion, the present study of three carriers of Y-autosome translocations highlights the importance of chromosomal rearrangement and position effect of susceptibility genes, and may help to improve genetic counseling in male infertility therapy.

\section{Acknowledgements}

The present study is supported by Guangdong Provincial Natural Science Foundation (grant no. S2013040012649) the New Teacher Project of Education Ministry (grant no. 20134423120005), the National Natural Science Funds of China (grant nos. 81202604 and 81401205) and the Scientific Research Project of Guangzhou Science and Information Bureau (grant no. 2014J4100024).

\section{References}

1. Giltay JC, Tiemessen CH, van Inzen WG and Scheres JM: One normal child and a chromosomally balanced/normal twin after intracytoplasmic sperm injection in a male with a de-novo $\mathrm{t}(\mathrm{Y} ; 16)$ translocation. Hum Reprod 13: 2745-2747, 1998

2. Barasc H, Mary N, Letron R, Calgaro A, Dudez AM, Bonnet N, Lahbib-Mansais Y, Yerle M, Ducos A and Pinton A: Y-autosome translocation interferes with meiotic sex inactivation and expression of autosomal genes: A case study in the pig. Sex Dev 6: 143-150, 2012.

3. Reijo R, Lee TY, Salo P, Alagappan R, Brown LG, Rosenberg M, Rozen S, Jaffe T, Straus D, Hovatta O, et al: Diverse spermatogenic defects in humans caused by $\mathrm{Y}$ chromosome deletions encompassing a novel RNA-binding protein gene. Nat Genet 10: $383-393,1995$.
4. van der Ven $\mathrm{K}$, Messer $\mathrm{L}$, van der Ven $\mathrm{H}$, Jeyendran $\mathrm{RS}$ and Ober C: Cystic fibrosis mutation screening in healthy men with reduced sperm quality. Hum Reprod 11: 513-517, 1996.

5. Jiang YT, Zhang HG, Wang RX, Yu Y, Zhang ZH and Liu RZ: Novel $Y$ chromosome breakpoint in an infertile male with a de novo translocation $\mathrm{t}(\mathrm{Y} ; 16)$ : A case report. J Assist Reprod Genet 29: 1427-1430, 2012.

6. Martin RH: Cytogenetic determinants of male fertility. Hum Reprod Update 14: 379-390, 2008.

7. Schmid M: Demonstration of Y/autosomal translocations using distamycin A. Hum Genet 53: 107-109, 1979.

8. Nielsen J and Rasmussen K: Y/autosomal translocations. Clin Genet 9: 609-617, 1976.

9. Chen CP, Lin SP, Tsai FJ, Wang TH, Chern SR and Wang W: Characterization of a de novo unbalanced Y;autosome translocation in a $45, X$ mentally retarded male and literature review. Fertil Steril 90: 1198.e11-e18, 2008

10. Chen CP, Fu CH, Chern SR, Wu PS, Su JW, Lee CC, Lee MS and Wang W: De novo unbalanced translocation resulting in monosomy for distal $5 \mathrm{p}(5 \mathrm{p} 14.1 \rightarrow$ pter $)$ and $14 \mathrm{q}(14 \mathrm{q} 32.31 \rightarrow$ qter $)$ associated with fetal nuchal edema, microcephaly, intrauterine growth restriction and single umbilical artery: Prenatal diagnosis and molecular cytogenetic characterization. Taiwan J Obstet Gynecol 52: 401-406, 2013

11. Chen-Shtoyerman R, Josefsberg Ben-Yehoshua S, Nissani R, Rosensaft J and Appelman Z: A prevalent Y;15 translocation in the Ethiopian Beta Israel community in Israel. Cytogenet Genome Res 136: 171-174, 2012.

12. Dutrillaux B, Descailleaux J, Viegas-Pequignot E and Couturier J: Y-autosome translocation in Cacajao calvus rubicundus (Platyrrhini). Ann Genet 24: 197-201, 1981.

13. Sasagawa I, Nakada T, Adachi Y, Kato T, Sawamura T, Ishigooka $\mathrm{M}$ and Hashimoto T: Y-autosome translocation associated with azoospermia. Scand J Urol Nephrol 27: 285-268, 1993.

14. Cui YX, Xia XY, Pan LJ, Wang YH, Yao B and Huang YF: An infertile male with apparent 45, X turned out to have 45,X,der(Y) $\mathrm{t}(\mathrm{Y} ; 13)(\mathrm{q} 11.2 ; \mathrm{q} 12),-13$ : Clinicopathologic and cytogenomic studies. Fertil Steril 88: 1676.e7-e11, 2007.

15. Alves C, Carvalho F, Cremades N, Sousa M and Barros A: Unique (Y;13) translocation in a male with oligozoospermia: Cytogenetic and molecular studies. Eur J Hum Genet 10: 467-474, 2002.

16. Viguie F, Romani F and Dadoune JP: Male infertility in a case of $(Y ; 6)$ balanced reciprocal translocation. Mitotic and meiotic study. Hum Genet 62: 225-227, 1982.

17. Delobel B, Djlelati R, Gabriel-Robez O, Croquette MF, Rousseaux-Prevost R, Rousseaux J, Rigot JM and Rumpler Y: Y-autosome translocation and infertility: Usefulness of molecular, cytogenetic and meiotic studies. Hum Genet 102: 98-102, 1998.

18. Moreau N, Teyssier M and Rollet J: A new case of (Y;1) balanced reciprocal translocation in an infertile man with Hodgkin's disease. J Med Genet 24: 379-380, 1987.

19. Yumura Y, Murase M, Katayama K, Segino M, Aizawa Y, Kuroda SN and Noguchi K: Y-autosome translocation associated with male infertility: A case report. Hinyokika Kiyo 58: 307-310, 2012.

20. Vogt PH, Edelmann A, Kirsch S, Henegariu O, Hirschmann P, Kiesewetter F, Köhn FM, Schill WB, Farah S, Ramos C, et al: Human Y chromosome azoospermia factors (AZF) mapped to different subregions in Yq11. Hum Mol Genet 5: 933-943, 1996.

21. Krausz C, Forti G and McElreavey K: The Y chromosome and male fertility and infertility. Int J Androl 26: 70-75, 2003.

22. Krausz C, Chianese C, Giachini C, Guarducci E, Laface I and Forti G: The Y chromosome-linked copy number variations and male fertility. J Endocrinol Invest 34: 376-382, 2011.

23. Vogt PH: Genomic heterogeneity and instability of the AZF locus on the human Y chromosome. Mol Cell Endocrinol 224: $1-9,2004$ 